\title{
Geschwindigkeitsbegrenzungen: wirksame Prophylaxe gegen Unfallfolgen
}

\begin{abstract}
Die Einführung von innerstädtischen Geschwindigkeitsbegrenzungen (20 $\mathrm{mph}=32 \mathrm{~km} / \mathrm{h}$ ) in London hat zu einem beträchtlichen Rückgang von Verletzungen und Todesfällen durch Verkehrsunfälle geführt.
\end{abstract}

- Seit 1987 wurden in London Geschwindigkeitsbegrenzungen bis 20 mph auf einer Länge von insgesamt $1263 \mathrm{~km}$ eingeführt. Seither nahmen in diesen 119029 Straßensegmenten Unfälle um $41,9 \% \mathrm{ab}$, bei Kindern unter 15 Jahren sogar um 48,5\%. Auch schwere Verletzungen und Todesfälle gingen um 46,3\% zurück, wiederum mit 50,2\% stärker bei Kindern. Da im genannten Zeitraum auch in benachbarten Straßen ohne Geschwindigkeitsbegrenzung Unfälle und ihre Folgen um 5,4 bis 9,7\% sanken, hat sich der Verkehr nicht verlagert und andernorts zu mehr Unfällen geführt.

Im Zeitraum zwischen 1986 und 2006 sind in ganz London Verkehrsunfälle seltener geworden. Wenn dieser Zeittrend und die Dauer der Verkehrsänderungen in den einzelnen

\begin{tabular}{|c|c|c|c|}
\hline \multicolumn{4}{|c|}{ Weniger Tote und Verletzte } \\
\hline & \multicolumn{3}{|c|}{$\begin{array}{l}\text { Abnahme (\%) ohne/mit } \\
\text { Geschwindigkeitsbegrenzung }\end{array}$} \\
\hline & Ohne & $20 \mathrm{mph}(32 \mathrm{~km} / \mathrm{h})$ & Jährlich \\
\hline Alle Unfälle & 8,0 & 41,9 & 1,7 \\
\hline Unfälle bei Kindern & 9,7 & 48,5 & 3,4 \\
\hline $\begin{array}{l}\text { Schwere Verletzungen } \\
\text { und Todesfälle }\end{array}$ & 5,4 & 50,2 & 5,2 \\
\hline
\end{tabular}

Straßen berücksichtigt wurde, errechnet sich ein jährlicher Rückgang der Unfälle um 1,7\%, bei Kindern um $3,4 \%$ und bei den schweren Verletzungen und Todesfällen um 5,2\% (s. Tab. 1).

KOMMENTAR: Experten betrachten überhöhte und unangemessene Geschwindigkeit von Kraftfahrzeugen als die bedeutendste Ursache für Verkehrsunfälle. Für die Autoinsassen ist die Gefahr durch mancherlei Maßnahmen wie besser konstruierte Fahrzeuge, Sicherheitsgurte

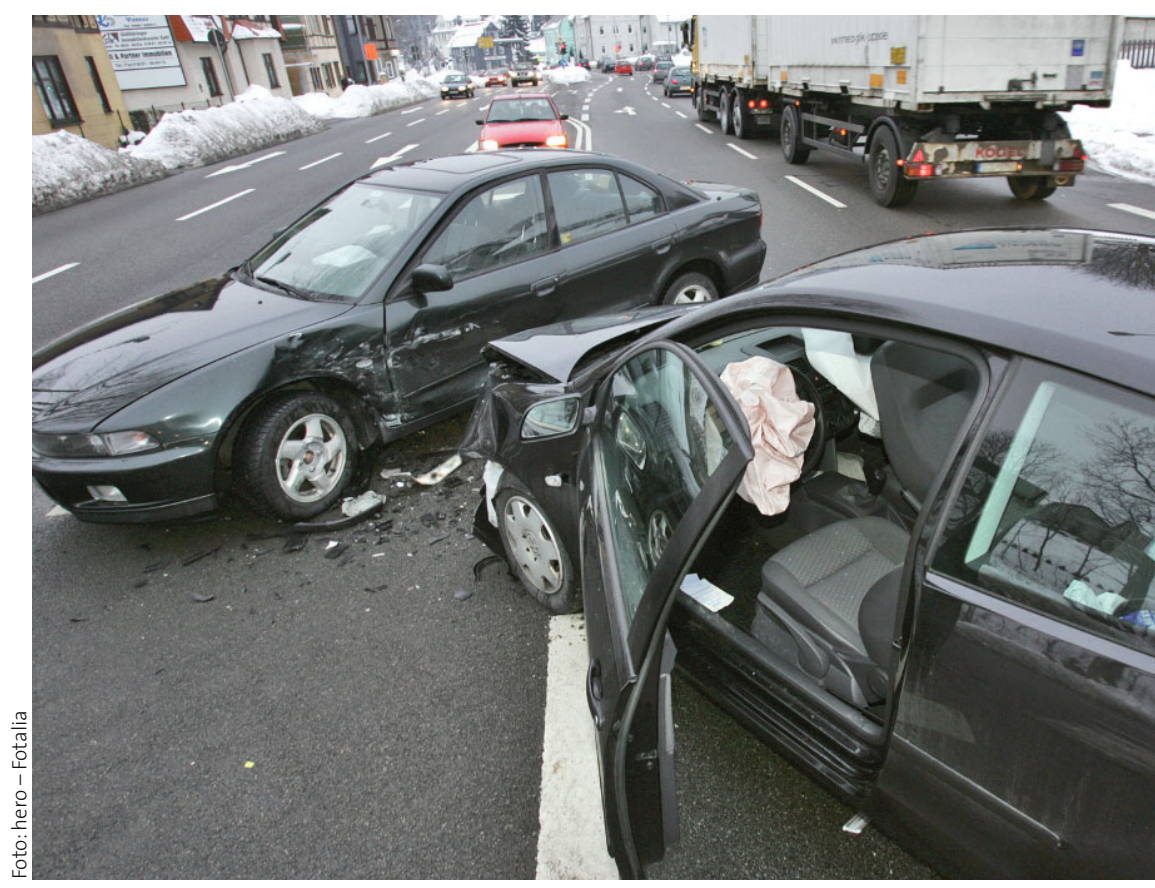

und Airbags deutlich reduziert, während für Fußgänger - insbesondere Kinder und Zweiradfahrer vergleichsweise wenig Vorsorge getroffen wurde. Diese Verkehrsteilnehmer können vor allem durch Geschwindigkeitsbegrenzungen geschützt werden.

Eine konservative Hochrechnung der Autoren ergibt, dass in London durch die bisherigen Geschwindigkeitsbegrenzungen jährlich 203 Unfälle mit 27 schweren Verletzungen verhindert wurden. Wenn diese Maßnahme auf alle kleineren Straßen und Wohngebiete der Stadt ausgedehnt würden, könnten allein in London jährlich 692 Unfälle mit 100 Todesfällen und schweren Verletzungen vermieden werden. Geschwindigkeitsbegrenzung ist eine "nebenwirkungsfreie Prophylaxe" gegen Unfallfolgen mit einer Wirksamkeit, die man sich für manche Erkrankungen wünschen würde.

H. HolzG ReVE -

\footnotetext{
-C. Grundy et al.

Effect of $20 \mathrm{mph}$ traffic speed zones on road injuries in London, 1986-2006: controlled interrupted time series analysis. Brit. Med. J. 340 (2010) 31
}

4 Hier kommt jede Geschwindigkeitsbegrenzung zu spät. 